\title{
Neuroendocrine differentiation in castration-resistant prostate cancer: A case report
}

\author{
DIMITRIOS PRIFTAKIS $^{1 *}$, NIKOLAOS KRITIKOS ${ }^{1 *}$, STAVROS STAVRINIDES $^{2 *}$, \\ STEFANOS KLEANTHOUS ${ }^{1 *}$ and NIKOLAOS BAZIOTIS ${ }^{1 *}$
}

Departments of ${ }^{1}$ Nuclear Medicine and ${ }^{2}$ Gastroenterology, St. Savvas Anticancer-Oncology Hospital, 11522 Athens, Greece

Received June 25, 2015; Accepted July 22, 2015

DOI: $10.3892 / \mathrm{mco} .2015 .645$

\begin{abstract}
The most common type of prostate cancer is acinar adenocarcinoma, which is androgen-dependent and, therefore, treated with chemical or surgical castration and androgen receptor inhibition. However, the disease usually progresses to castration-resistant prostate cancer (CRPC). A neuroendocrine pattern is frequently observed in the cellular composition of CRPC, which is considered to emerge as an effect of androgen deprivation therapy. This is the case report of a 69-year-old patient with prostate adenocarcinoma, who, after an initial period of disease control with radiotherapy and antiandrogens, was diagnosed with CRPC with high levels of prostate-specific antigen (PSA), unresponsive to androgen inhibition, with accompanying lung and osseous metastases. Bronchial biopsy of the lung metastasis revealed infiltration by non-small-cell adenocarcinoma of prostatic origin with neuroendocrine characteristics. On somatostatin receptor scintigraphy with ${ }^{99} \mathrm{~m}$ Tc-octreotide, there was high uptake by almost all known lung and osseous metastases. The patient was subsequently treated with a combination of docetaxel and octreotide, and a partial response was observed 6 months later, with reduction of the PSA level and the size of the lung metastasis. The aim of the present study was to provide a clinical example of the previously demonstrated, in vitro and in vivo, synergistic antitumor activities of docetaxel and octreotide in cases of CRPC selected by means of histological confirmation of their neuroendocrine nature and somatostatin receptor scintigraphy.
\end{abstract}

Correspondence to: Dr Dimitrios Priftakis, Department of Nuclear Medicine, St. Savvas Anticancer-Oncology Hospital, 171 Alexandras Avenue, 11522 Athens, Greece

E-mail: d84priftakis@gmail.com

${ }^{*}$ Contributed equally

Key words: castration-resistant, prostate cancer, neuroendocrine transdifferentiation, docetaxel, octreotide, somatostatin analogues, somatostatin receptor scintigraphy

\section{Introduction}

Prostate cancer is a very common type of cancer, accounting for $1 / 4$ of all cancer cases in men, and is the second most common cause of cancer-related mortality among men (1).

At least $90 \%$ of prostate cancers are initially diagnosed as acinar adenocarcinomas (2), which are almost always androgen-dependent (3). This reliance of the development of prostatic adenocarcinoma on androgens working through the androgen receptor (AR), is the basis for the use of chemical or surgical castration and AR inhibition as standard therapies (3). However, after an initial period of disease control through targeting the androgen axis, the disease almost inevitably progresses to castration-resistant prostate cancer (CRPC) (4).

A neuroendocrine pattern is frequently observed in the cellular composition of CRPC, which was not present in the initial diagnosis (5). The emergence of this neuroendocrine pattern in CRPC has been attributed to the effect of androgen deprivation therapy $(3,4,6)$ and two main mechanisms have been hypothesized. The first hypothesis suggests that, under prolonged hormonal manipulation, the resistant neuroendocrine-like tumor cell populations are selected from an initially heterogeneous tumor (3). The second hypothesis suggests that prolonged androgen deprivation may activate a process referred to as neuroendocrine transdifferentiation, which enables prostatic adenocarcinoma cells to acquire neuroendocrine characteristics (4).

There remains the question of whether the assessment of the neuroendocrine pattern in CRPC may be useful in the selection of potential responders to target therapies, such as somatostatin analogues (5), as in the present case.

\section{Case report}

A 69-year-old man was initially diagnosed with Gleason 4+4 prostate adenocarcinoma in 2007, and was treated by radiotherapy along with the antiandrogen bicalutamide and the gonadotrophin-releasing hormone agonist triptorelin. In 2009, the patient underwent nephrectomy due to severe trauma of the left kidney during an accident. Between 2009 and 2013, the patient was intermittently treated with bicalutamide, degarelix and other luteinizing hormone-releasing hormone (LHRH) agonists, due to responsive biochemical recurrence with mild elevation of the prostate-specific antigen (PSA) levels, the 


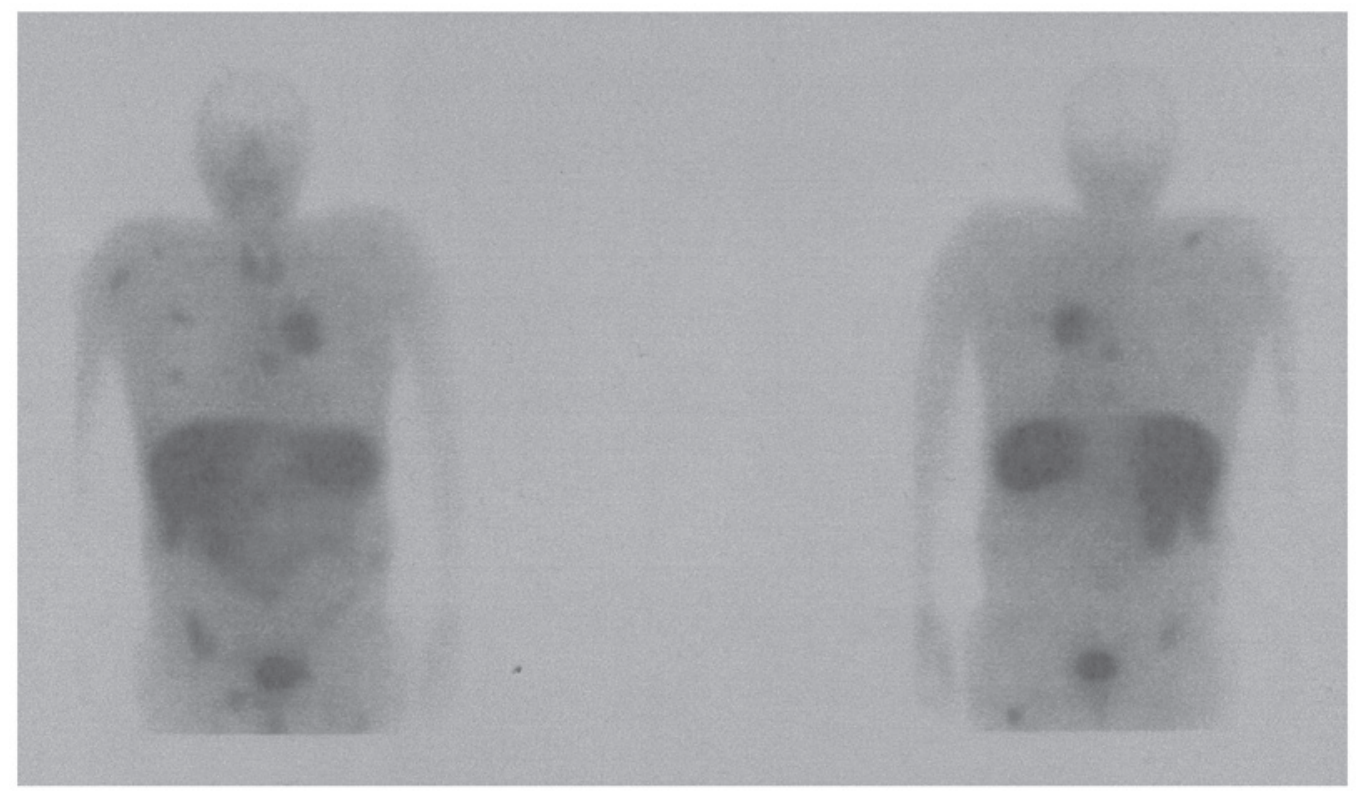

Figure 1. Anterior (left) and posterior (right) view of somatostatin receptor imaging with ${ }^{99 \mathrm{~m} T c-o c t r e o t i d e . ~ H i g h ~ u p t a k e ~ o f ~ t h e ~ r a d i o p h a r m a c e u t i c a l ~ w a s ~}$ observed by the known lung metastasis and the osseous metastases of the spine, the anterior 6th rib, the right scapula and the left femur, as well as in other locations, such as the right ilium, the right clavicle and the right humerus.

highest being $2.2 \mathrm{ng} / \mathrm{ml}$. In the 3 months from October, 2013 to January, 2014, there was an elevation of PSA from 3.49 to $22.14 \mathrm{ng} / \mathrm{ml}$, despite ongoing treatment with LHRH agonists. The patient also complained of dyspnea and chest computed tomography (CT) revealed a $7.5-\mathrm{cm}$ tumor in the upper lobe of the left lung, which caused stenosis of the respective bronchus. There were also enlarged mediastinal lymph nodes, with a maximum diameter of $17 \mathrm{~mm}$. Skeletal radionuclide imaging with ${ }^{99 \mathrm{~m}} \mathrm{Tc}$-methylene diphosphonate also revealed numerous osseous metastases in the thoracic and lumbar spine, the anterior 6th right rib, the right scapula and the left femur. Bronchial biopsy of the lung metastasis revealed infiltration by non-small-cell adenocarcinoma of prostatic origin, with a small proportion of CD56- and synaptophysin-positive cells, indicating neuroendocrine differentiation. The Ki-67 index was $40 \%$. In somatostatin receptor scintigraphy with ${ }^{99 \mathrm{~m}} \mathrm{Tc}$-octreotide, there was high uptake in various locations, including almost all known lung and osseous metastases (Fig. 1), thus confirming a global neuroendocrine differentiation of the recurrent metastatic disease. According to these findings, the selected treatment consisted of chemotherapy with docetaxel and octreotide injections. In the follow-up chest CT 6 months later, the size of the lung metastasis had been reduced to $4.7 \mathrm{~cm}$, there were no enlarged mediastinal lymph nodes, while the PSA level had decreased to $3.09 \mathrm{ng} / \mathrm{ml}$.

\section{Discussion}

According to the American Society of Clinical Oncology and the Cancer Care Ontario clinical practice guideline, the main option for patients with metastatic CRPC is systemic therapy with docetaxel, with moderate benefit, which is however supported by strong evidence (7). Additionally, the guideline suggests continuation of androgen deprivation indefinitely (7). Another viable option may be the therapeutic targeting of the somatostatin receptors in the surface of the neuroendocrine tumor cells with a somatostatin analogue. This option is supported by the relatively limited but persuasive literature, to which we aim to add our own experience (8-10).

Somatostatin receptor scintigraphy with ${ }^{111}$ In-pentetreotide (Octreoscan) or with ${ }^{99 \mathrm{~m}} \mathrm{Tc}$-labelled octreotide analogue is mandatory to establish the adequate presence of somatostatin receptors in the metastatic lesions (11). The first to identify somatostatin receptors in the metastatic lesions of CRPC patients were Nilsson et al (12) in 1995, who also implemented the first therapy with octreotide in a patient with CRPC and bone metastases. The result of this therapeutic intervention was a reduction in the metabolic activity of a major bone metastasis on ${ }^{11} \mathrm{C}$-methionine positron emission tomography, accompanied by symptomatic benefit in the form of pain relief.

Somatostatin receptor scintigraphy is only used to assess the feasibility of somatostatin analogue therapy and should not be used for diagnosis or as a surrogate of histological confirmation of neuroendocrine differentiation, since it has been found to be positive in $37 \%$ of patients with CRPC, with only $11 \%$ exhibiting bone metastases and only $15 \%$ visceral metastases on Octreoscan (11). In particular, when a lung tumor is identified, as in our case, histological confirmation of the prostatic origin of the lesion is crucial, as somatostatin receptor scintigraphy is usually positive in non-neuroendocrine lung cancers, such as non-small-cell lung cancer $(13,14)$.

The antitumor effects of somatostatin and its analogues include inhibition of cell proliferation, invasion and tumor angiogenesis, and induction of apoptosis through complex pathways mediated by the somatostatin receptor subtypes on tumor cells and on cells in their microenvironment (8). Specifically, in prostate cancer, somatostatin analogues appear to exert a limited effect as monotherapy (9). However, the combination of somatostatin analogues with various chemotherapeutic and other agents has been investigated in clinical studies, with 
favorable results in terms of progression-free survival with the addition of the somatostatin analogue (9). Due to the limited number of patients in those studies, further randomized studies, including a higher number of patients, are required to demonstrate the role of somatostatin analogue-containing combination regimens in prostate cancer (9).

Recently, the synergistic antitumor activities of docetaxel and octreotide, the regimen that was administered to our patient, were demonstrated in vitro in a study by Zhu et al (10). That study demonstrated that the combination of docetaxel and octreotide was more efficient in inducing apoptosis and reducing migration compared with either drug alone, and that the addition of octreotide increased docetaxel sensitivity and cytotoxicity (10).

In conclusion, the results presented above, also supported by other studies on the molecular mechanism of the docetaxel-octreotide combination (15), suggest that, in carefully selected cases, somatostatin analogues such as octreotide may be added to standard chemotherapy with docetaxel. The present study also recommends that the selection of the cases includes histological confirmation of the neuroendocrine nature of CRPC, as well as somatostatin receptor scintigraphy. The outcome of this approach in our case prompts further investigation in this direction.

\section{References}

1. Siegel RL, Miller KD and Jemal A: Cancer statistics, 2015. CA Cancer J Clin 65: 5-29, 2015.

2. Humphrey PA: Histological variants of prostatic carcinoma and their significance. Histopathology 60: 59-74, 2012.

3 . Terry S and Beltran H: The many faces of neuroendocrine differentiation in prostate cancer progression. Front Oncol 4: 60, 2014

4. Nouri M, Ratther E, Stylianou N, Nelson CC, Hollier BG and Williams ED: Androgen-targeted therapy-induced epithelial mesenchymal plasticity and neuroendocrine transdifferentiation in prostate cancer: An opportunity for intervention. Front Oncol 4: 370, 2014.

5. Matei DV, Renne G, Pimentel M, Sandri MT, Zorzino L, Botteri E, De Cicco C, Musi G, Brescia A, Mazzoleni F, et al: Neuroendocrine differentiation in castration-resistant prostate cancer: A systematic diagnostic attempt. Clin Genitourin Cancer 10: 164-173, 2012.
6. Beltran H, Tagawa ST, Park K, MacDonald T, Milowsky MI, Mosquera JM, Rubin MA and Nanus DM: Challenges in recognizing treatment-related neuroendocrine prostate cancer. J Clin Oncol 30: e386-e389, 2012.

7. Basch E, Loblaw DA, Oliver TK, Carducci M, Chen RC, Frame JN, Garrels K, Hotte S, Kattan MW, Raghavan D, et al: Systemic therapy in men with metastatic castration-resistant prostate cancer: American Society of Clinical Oncology and Cancer Care Ontario clinical practice guideline. J Clin Oncol 32: 3436-3448, 2014.

8. Bousquet C, Lasfargues C, Chalabi M, Billah SM, Susini C, Vezzosi D, Caron P and Pyronnet S: Clinical review: Current scientific rationale for the use of somatostatin analogs and mTOR inhibitors in neuroendocrine tumor therapy. J Clin Endocrinol Metab 97: 727-737, 2012.

9. Keskin O and Yalcin S: A review of the use of somatostatin analogs in oncology. Onco Targets Ther 6: 471-483, 2013.

10. Zhu S, Oremo JA, Li S, Zhen M, Tang Y and Du Y: Synergistic antitumor activities of docetaxel and octreotide associated with apoptotic-upregulation in castration-resistant prostate cancer. PLoS One 9: e91817, 2014.

11. Mencobonii M, Tredici S, Rebella L, Bergaglio M, Galbusera V, Manzara A, Claudiani F, Malcangi B and Varaldo M: Effect of chemotherapy on somatostatin receptor detection with octreotide scintigraphy in hormone-refractory prostate cancer patients. Anticancer Res 26: 2233-2235, 2006.

12. Nilsson S, Reubi JC, Kalkner KM, Laissue JA, Horisberger U, Olerud C and Westlin JE: Metastatic hormone-refractory prostatic adenocarcinoma expresses somatostatin receptors and is visualized in vivo by $\left({ }^{111} \mathrm{In}\right)$-labeled DTPA-D-(Phe $)$-octreotide scintigraphy. Cancer Res 55 (Suppl 23): 5805s-5810s, 1995.

13. Krenning EP, Kwekkeboom DJ, Bakker WH, Breeman WA, Kooij PP, Oei HY, van Hagen M, Postema PT, de Jong M, Reubi JC, et al: Somatostatin receptor scintigraphy with $\left({ }^{111}\right.$ In-DTPA-D-Phe $\left.{ }^{1}\right)$-and $\left({ }^{123}{ }^{12}-T_{y}{ }^{3}\right)$-octreotide: The Rotterdam experience with more than 1,000 patients. Eur J Nucl Med 20: 716-731, 1993.

14. Herlin G, Kölbeck KG, Menzel PL, Svensson L, Aspelin P, Capitanio A and Axelsson R: Quantitative assessment of ${ }^{99 \mathrm{~m} T c}$-depreotide uptake in patients with non-small-cell lung cancer: Immunohistochemical correlations. Acta Radiol 50: 902-908, 2009.

15. Lattanzio L, Tonissi F, Monteverde M, Milano G, Merlano MC and Lo Nigro C: Differential molecular mechanism of docetaxel-octreotide combined treatment according to the docetaxel-resistance status in PC3 prostate cancer cells. Anticancer Drugs 24: 120-130, 2013. 